\title{
Transformación de Roxarsona por un consorcio bacteriano aislado desde suelo en condiciones Aeróbicas
}

\section{Transformation of Roxarsone by bacterial consortium Isolated from soil in aerobic condition}

\author{
Víctor Guzmán-Fierro ${ }^{1}$, Marcela Pedraza 1 , Carla León ${ }^{1}$, Víctor L Campos ${ }^{1 *} \&$ María-Angélica \\ MONDACA $^{1}$
}

${ }^{1}$ Laboratorio de Microbiología Ambiental, Departamento de Microbiología, Facultad de Ciencias Biológicas. Universidad de Concepción. P.O. Box 152-C. Concepción, Chile.

*E-mail:vcampos@udec.cl

\begin{abstract}
RESUMEN
Roxarsone (ROX) es un compuesto organoarsenical utilizado como aditivo alimentario en la industria avicola y tiene el riesgo potencial de contaminar el ambiente, debido al uso de estiércol de aves de corral como fertilizante. El objetivo del presente trabajo fue aislar y caracterizar un consorcio bacteriano, proveniente de un suelo agricola, capaz de degradar ROX bajo condiciones aeróbicas. Muestras de suelos colectadas en las cercanías de una industria avícola (Octava región, CHILE) fueron enriquecidas en caldo R2A 9,1\% (p/v). El consorcio bacteriano seleccionado fue crecido a $25^{\circ} \mathrm{C}$ con agitación constante $(125 \mathrm{rpm})$ en ausencia y presencia de $\operatorname{ROX}(0,5 \mathrm{mM})$ y en condiciones de aerobiosis. El consorcio fue caracterizado molecularmente por DGGE acoplado a un análisis de escalamiento multidimensional (MDS) y mediante la determinación de los índices de diversidad de Shannon-Weaver $\left(H^{\prime}\right)$ y de equidad de Pielou $\left(J^{\prime}\right)$. Además, se evaluó el efecto de factores abióticos (temperatura, $\mathrm{pH}$ y fuentes carbono) sobre la degradación bacteriana de ROX en un medio químicamente definido (MQD). Los resultados demostraron que la velocidad de crecimiento del consorcio bacteriano en presencia de ROX fue 1,38 veces mayor respecto al control sin ROX, degradándose hasta un 41,6\% del organoarsenical luego de 168 h de incubación. El análisis MDS reveló que la estructura del consorcio cambió de 70\% a 50\% su similitud en presencia de ROX, mientras que se observó una disminución y aumento en los índices de $H^{\prime}$ y $J^{\prime}$, respectivamente, en presencia de ROX. Los factores abióticos mostraron que, luego de $120 \mathrm{~h}$ de incubación, a $37^{\circ} \mathrm{C} \mathrm{y} \mathrm{pH} 7$, en presencia de lactato como fuente de carbono la reducción ROX llegó a 57,8\% y en presencia de glucosa la biotransformación ROX alcanzó 62,3\%, ambas superiores a otras fuentes de carbono utilizadas. La degradación microbiológica de ROX en condiciones aeróbicas ha sido pobremente informado y tiene un potencial impacto ambiental sobre suelos agrícolas fertilizados con estiércol contaminado ROX.
\end{abstract}

Palabras claves: arsénico, roxarsona, órgano-arsenical, consorcio bacteriano.

\begin{abstract}
Roxarsone (ROX), an organoarsenical compound used as food additive in the poultry industry, has the potential risk to contaminate the environment, mainly by the use of poultry industry manure as fertilizer. The aim of this work was to isolate and characterize a bacterial consortium from agricultural soil, capable of degrading ROX under aerobic conditions. Soil samples were collected from a poultry industry (Bio Bio Region, Chile) and were enriched in 9.1\% (weight/volume) R2A broth at room temperature. The selected bacterial consortium was grown in the absence and presence of $0.5 \mathrm{mM} \mathrm{ROX}$ at $25{ }^{\circ} \mathrm{C}$ with agitation $(125 \mathrm{rpm})$ and under aerobic conditions. The consortium was characterized by DGGE coupled to a multidimensional scaling analysis (MDS). Shannon-Weaver $\left(H^{\prime}\right)$ and Pielou $\left(J^{\prime}\right)$ indexes were determined. In addition, effects of abiotic factors (temperature and $\mathrm{pH}$ ) and carbon sources on the transformation of ROX were evaluated in a chemically defined medium (CDM). The results showed that the growth rate was 1.38 -fold higher in ROX presence and that ROX degradation was $41.6 \%$ after $168 \mathrm{~h}$ of incubation. MDS results demonstrated that the consortium structure changes from $70 \%$ to $50 \%$ similarity while a decrease and increase in indexes of $H^{\prime}$ and $J$ ', respectively, was observed in the presence of ROX. Abiotic factors assessment showed that after $120 \mathrm{~h}$ incubation, at $37^{\circ} \mathrm{C}$ and $\mathrm{pH} 7$, when using lactate as carbon source ROX reduction reached $57.8 \%$ but when glucose was used ROX biotransformation reached $62.3 \%$, exceeding other carbon sources used. Microbiological degradation of ROX under aerobic conditions has been rarely reported and it has a potential environmental impact on agricultural soils fertilized with ROX contaminated manure.
\end{abstract}

KEYWORDS: arsenic, roxarsone, organoarsenic, bacterial consortium. 


\section{INTRODUCCIÓN}

Roxarsona (ácido 3-Nitro-4-hidroxifenilarsénico) es un compuesto organoarsenical utilizado en la industria avícola como aditivo alimenticio para promover crecimiento y controlar parásitos intestinales(coccidios). Aproximadamente el $88 \%$ de los pollos de engorde producidos en Estados Unidos (EE.UU) son alimentados con roxarsona (Nachman et al. 2012), que se excreta casi principalmente inalterada en el estiércol. Cerca de 900 toneladas métricas de roxarsona se liberan anualmente en el medio ambiente en los EE.UU. (Garbarino et al. 2003), cuyo contenido de arsénico oscila entre 14 y $76 \mathrm{mg} \mathrm{kg}^{-1}$ (Arai et al. 2003; Jackson \& Bertsch 2001; Jackson et al. 2003).

El estiércol al poseer una gran cantidad de nutrientes es utilizado principalmente como fertilizante. Durante su aplicación, la roxarsona $(\mathrm{ROX})$ es rápidamente degradada a arsénico inorgánico, como arseniato (As V) y arsenito (As III), así como una variedad de compuestos organoarsenicales (Silbergeld \& Nachman 2008). Dependiendo del nivel de humedad de los suelos, cuando As (V) se encuentra en un entorno pobre en oxígeno, puede ser fácilmente transformado microbianamente a As (III) o dimetilarsenato, que pueden movilizarse o ser absorbidos rápidamente por los componentes del suelo (Nachman et al. 2005).

Existen pocos reportes sobre el aislamiento y la identificación de los microorganismos involucrados en la transformación aeróbica de roxarsona. Los estudios realizados por Stolz et al. (2007) demostraron que las especies de Clostridium presentes en el estiércol de aves de corral, transforman roxarsona en arseniato en condiciones anaerobias. Por otro lado, Garbarino et al. (2003) llevaron a cabo experimentos que demostraron la biotransformación de roxarsona a arsénico inorgánico y otros metabolitos, pero no aislaron ni caracterizaron los microorganismos implicados.

Considerando que la aplicación de composta se aplica en los primeros centímetros de los suelos, un ambiente esencialmente óxico donde la disponibilidad y movilidad de las especies tóxicas del arsénico se incrementaría producto del metabolismo aeróbico de las comunidades microbianas nativas, es importante destacar que no existen antecedentes previos acerca de la interacción entre las comunidades bacterianas aeróbicas de suelo y la roxarsona (ROX). Por lo tanto, el objetivo de este trabajo fue aislar y caracterizar un consorcio bacteriano, proveniente de un suelo agricola, capaz de degradar roxarsona bajo condiciones aeróbicas.

\section{MATERIALES Y MÉTODOS}

RECOLECCIÓN DE MUESTRAS

Se obtuvieron muestras de suelo agricola, fertilizado con estiércol de ave de corral, desde las cercanías de una industria avícola (Región de Bio Bio, CHILE). Se colectaron muestras de la superficie del suelo $(0 \mathrm{~cm})$ y se almacenaron a $4{ }^{\circ} \mathrm{C}$ hasta su posterior análisis.

ENRIQUECIMIENTO DE BACTERIAS PRESENTE EN MUESTRAS DE SUELO

Las muestras de suelo fueron incubadas por 10 días en caldo R2A 9,1\% (R2A/2) con agitación (125 rpm) a temperatura ambiente y en condiciones aeróbicas.

DEGRADACIÓN BACTERIANA DE ROXARSONA (ROX)

A partir de los inóculos obtenidos en el enriquecimiento, se traspasaron $5 \mathrm{ml}$ de cada uno de ellos a $100 \mathrm{ml}$ de medio $\mathrm{R} 2 \mathrm{~A} / 2$ adicionados con $\mathrm{ROX}(0,5 \mathrm{mM})$. Como control negativo se utilizó caldo R2A/2 adicionado exclusivamente con $\operatorname{ROX}(0,5 \mathrm{mM})$. La incubación se realizó durante 168 $\mathrm{h}$ con toma de muestra cada 4 y $12 \mathrm{~h}$. Se filtraron $(0,22 \mu \mathrm{m}$ Millipore) alícuotas de $1 \mathrm{ml}$ tanto del consorcio bacteriano adicionado con ROX como del control negativo, para luego transferir desde cada cultivo $200 \mu \mathrm{l}$ en triplicado a una microplaca de 96 pocillos. La detección de ROX se realizó en un espectrofotómetro (EpochTM, BIOTEK $®$ ) y los análisis de degradación se realizaron integrando de los espectrogramas $(310 \mathrm{~nm}-500 \mathrm{~nm})$ a través del método de trapezoide, usando el software Gen5TM (BIOTEK () ). Las cinéticas de degradación obtenidas fueron analizadas a través del modelo de aproximación matemática Weibull (Zwietering et al. 1990), el cual entregó la velocidad de degradación de ROX.

Cinética de CRecimiento del CONSORCIO BaCteriano

El crecimiento celular fue analizado en cultivos con y sin ROX después de 168 h de incubación. Para ello se determinó la densidad óptica a $600 \mathrm{~nm}$ en muestras colectadas en triplicado desde cada cultivo utilizando un espectrofotómetro EpochTM (BIOTEK $囚)$. Las curvas obtenidas fueron analizadas a través de diferentes modelos de aproximación matemática como, Gompertz, Logistic, exponencial Mathusian, exponencial plateau y Weibull (Zwietering et al. 1990). Se optó por el modelo que mejor se ajustó a las cinéticas de crecimiento usando como criterio la prueba F de Fisher y la comparación de los coeficientes de determinación (R2). El modelo escogido fue utilizado para comparar las velocidades de crecimiento $(\mathrm{k})$ del consorcio bacteriano incubado en presencia y ausencia de ROX.

ExTRACCIÓN DE ADN, AMPLIFICACIÓN DEL GEN ADNR 16s y ANÁLISIS DGGE

El ADN total del consorcio bacteriano incubado $168 \mathrm{~h}$ en presencia y ausencia de ROX se extrajo utilizando el kit Ultra Clean ${ }^{\circledR}$ (MO BIO Laboratories, Inc.) siguiendo el protocolo proporcionado por el fabricante. El gen del ADNr-16S fue amplificado con los partidores universales EUB 9-27 y EUB 1542 (Brosius et al. 1978) utilizando los extractos de ADN 
total como templado. El cóctel de PCR fue ajustado a $50 \mu 1$ y contuvo los siguientes componentes: 12,5 $\mu \mathrm{l}$ EconoTaq ${ }^{\circledR}$ PLUS GREEN 2X Master Mix (Lucigen ${ }^{\circledR}$ Corporation), partidores EUB 9-27 $(1 \mu \mathrm{M})$ y EUB $1542 \mathrm{r}(1 \mu \mathrm{M}), 1 \mu \mathrm{l}$ de ADN templado y se ajustó al volumen final con agua DEPC (Dietilpirocarbonato). La PCR consistió de un paso de denaturación inicial a $94^{\circ} \mathrm{C}$ por 5 minutos seguido de 35 ciclos de amplificación (con cada ciclo programado a $94^{\circ} \mathrm{C}$ por 30 segundos, alineamiento a $55,6^{\circ} \mathrm{C}$ por 45 segundos y elongación a $72^{\circ} \mathrm{C}$ por 1,5 minutos) y una extensión final a $72^{\circ} \mathrm{C}$ por 10 minutos. Posteriormente se realizó un PCR anidado para la zona V3 utilizando los partidores $341 \mathrm{~F}$ y 534r enriquecido con 40 bases de GC (534r-GC) (Muyzer et al. 1993).

Esta segunda PCR se ajustó en un cóctel de $50 \mu \mathrm{l}$ que contuvo: 12,5 $\mu 1$ de EconoTaq ${ }^{\circledR}$ PLUS GREEN 2X Master Mix (Lucigen ${ }^{\circledR}$ Corporation), los partidores 341F (P3) $(1 \mu \mathrm{M})$ y $534 \mathrm{r}-\mathrm{GC}(\mathrm{P} 2)(1 \mu \mathrm{M}), 2 \mu \mathrm{l}$ de amplicón (del primer PCR) como templado y se ajustó al volumen final con agua DEPC (Dietilpirocarbonato). Se realizó un PCRTouchdown con las siguientes condiciones: un paso de denaturación inicial a $94^{\circ} \mathrm{C}$ por 5 minutos, luego 20 ciclos de; denaturación a $94^{\circ} \mathrm{C}$ por 30 segundos, alineamiento a $65^{\circ} \mathrm{C}$ por 45 segundos (la temperatura de alineamiento fue decreciendo $0,5^{\circ} \mathrm{C}$ en cada ciclo hasta llegar a $55^{\circ} \mathrm{C}$ ) y elongación a $72^{\circ} \mathrm{C}$; a continuación se realizaron 10 ciclos de; denaturación a $94^{\circ} \mathrm{C}$ por 30 segundos, alineamiento a $55^{\circ} \mathrm{C}$ por 45 segundos y elongación a $72^{\circ} \mathrm{C}$ por 1,5 minutos; para finalizar se realizó una extensión final de $72^{\circ} \mathrm{C}$ por 10 minutos. La concentración, pureza y tamaño de los productos PCR fueron chequeados mediante electroforesis en geles de agarosa $(1,5 \%)$ teñidos con el intercalante de ADN Gel Red (Biotium) (Campos et al. 2011).

La electroforesis en gel con gradiente denaturante (DGGE) se realizó con un sistema de DGGE 1001 (C.B.S Scientific Company Inc.). Se aplicaron $15 \mu 1$ de los productos de PCR de la región V3 (45 ng/ $\mu \mathrm{L})$ directamente sobre un gel de poliacrilamida al 6\% (peso/vol) en buffer TAE 1X (40 mM Tris, $20 \mathrm{mM}$ de acetato y $1 \mathrm{mM}$ EDTA) con gradiente denaturante de 20 a $80 \%$ (en donde $100 \%$ de denaturante contiene $7 \mathrm{M}$ de urea y $40 \%$ de formamida). Se realizó la electroforesis a un voltaje constante de 100 $\mathrm{V}$ a $60^{\circ} \mathrm{C}$ durante $18 \mathrm{~h}$. Después de la electroforesis, el gel se tiño durante 20 min con una solución $2 \mathrm{X}$ de SYBR Gold (Molecular Probes) de acuerdo a las especificaciones del fabricante, y se visualizó en un transiluminador (UVP Inc) (Campos et al. 2011). Los ensayos fueron realizados en duplicado.

\section{ANÁlisis del PERFIL DEL DGGE}

Se fotografiaron secciones ampliadas del gel de DGGE utilizando un sistema de imagen ChemImager 4000 (Alpha innotech). La intensidad de las bandas de DGGE (OTUs = unidades taxonómicas operacionales) fue determinada usando el software Gel-Pro Analyzer 4.0 (Applied Maths) donde una banda con una intensidad $\geq 5 \%$ de la banda más intensa del gel fue registrada como presente, mientras que una banda con una intensidad por debajo de este umbral fue considerada ausente. Los datos de presencia (1) o ausencia (0) de las bandas individuales en cada línea se utilizaron para construir una matriz binaria para la comparación de los perfiles de bandeo. Los datos binarios que representan a los patrones de bandas fueron utilizados para generar una matriz de distancia de comparación de pares (Leon et al. 2012). La matriz de distancia fue usada para construir un diagrama de escalamiento multidimensional (MDS) que representa un mapa de dos dimensiones con ejes artificiales $\mathrm{X}$ e $\mathrm{Y}$, donde cada muestra del DGGE corresponde a un punto de manera que muestras similares se disponen cercanas o juntas en el mapa. Los análisis de clustering y MDS fueron realizados con el software PRIMER V.6 (Clarke \& Gorley 2001).

Además, los OTUs fueron examinados utilizando el índice de diversidad de Shannon-Weaver $\left(H^{\prime}\right)$ (Shannon \& Weaver 1963) y el índice de equidad de Pielou ( $J^{\prime}$ ) (Pielou 1975). Se calcularon para cada muestra de la siguiente manera:

$$
\begin{aligned}
& H^{\prime}=-\sum(\mathrm{ni} / \mathrm{N}) \log (\mathrm{ni} / \mathrm{N}) \\
& J^{\prime}=\mathrm{H} / \log \mathrm{S}
\end{aligned}
$$

Donde ni es la intensidad relativa de cada banda del DGGE, $\mathrm{S}$ es el número de bandas del DGGE (utilizado para indicar el número de especies) y $\mathrm{N}$ es la suma de la intensidad relativa de todas las bandas (usado para estimar abundancia de especies) (Fromin et al. 2002).

EVALUACIÓN DE FACTORES ABIÓTICOS: TEMPERATURA, PH Y FUENTES DE CARBONO

Para evaluar el efecto de los factores abióticos en la transformación de ROX se utilizó el medio químicamente definido (MQD) descrito por Stolz et al. (2007). Los ensayos se realizaron traspasando alícuotas de $2,5 \mathrm{ml}$ del consorcio bacteriano enriquecido en $\mathrm{R} 2 \mathrm{~A} / 2$ a $30 \mathrm{ml}$ de $\mathrm{MQD}$, en cual fue adicionado con ROX $(0,5 \mathrm{mM})$. Como controles negativos se prepararon MQD adicionados sólo con ROX $(0,5 \mathrm{mM})$ bajo las diversas condiciones (Temperatura, $\mathrm{pH}$ y fuentes de carbono). El efecto de la temperatura de incubación fue evaluado a $15^{\circ} \mathrm{C}, 30^{\circ}$ y $37^{\circ} \mathrm{C}$. El efecto del $\mathrm{pH}$ fue evaluado ajustando las soluciones a $5.0,7.0 \mathrm{y}$ 9.0 (con $\mathrm{HCl} 0,01 \mathrm{M} \mathrm{o} \mathrm{NaOH} 0,01 \mathrm{M}$ ) y la temperatura de incubación que permitió mayor degradación de ROX por parte del consorcio bacteriano. Obtenidas las condiciones de temperatura y $\mathrm{pH}$ de máxima degradación de ROX, se evaluaron distintas fuentes de carbono tales como acetato, glucosa y lactato a una concentración final de $10 \mathrm{mM}$. Las estimaciones de degradación de ROX fueron realizadas a las $120 \mathrm{~h}$ de incubación integrando los espectrogramas (310 
nm-500 nm) a través del método de trapezoide mediante el software Gen5TM (BIOTEK $\left.{ }^{\circledR}\right)$. Por último, se calculó el porcentaje de degradación de ROX de la siguiente forma:

$\%$ Degradación $=\left(C_{i}-C_{f} C_{i}\right) \times 100$

Donde $\mathrm{C}_{\mathrm{i}}$ representa el área del espectrograma del filtrado

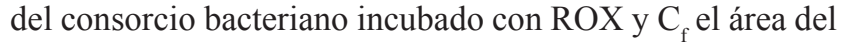
espectrograma para el control negativo.

\section{ANÁLISIS ESTADÍSTICO}

Se utilizó la prueba $\mathrm{t}$ de Student para comparar las velocidades de crecimiento $(\mathrm{k})$ en ausencia y presencia de ROX, además de los índices Shannon-Weaver y de equidad del DGGE para ayudar a caracterizar el consorcio bacteriano. Por otro lado, se evaluó la degradación de ROX frente a diferentes factores abióticos a través de un análisis de varianza (ANOVA) de una vía, y posteriormente, se seleccionó la prueba de Tukey para separar las medias. Para ambos análisis los valores de $\mathrm{P}<0,05$ fueron considerados como diferencia estadística, para lo cual se utilizó el programa MINITAB versión 15 (USA).

\section{RESULTADOS}

ANÁlisis DE DEGRADACIÓN DE ROXARSONA POR EL CONSORCIO BACTERIANO

Los análisis espectrofotométricos demostraron que el consorcio bacteriano tiene la capacidad de degradar en un $41,6 \%$ la $\operatorname{ROX}(0,5 \mathrm{mM})$ presente en el medio R2A/2, después de $168 \mathrm{~h}$ de incubación a $25^{\circ} \mathrm{C}$ y con agitación (Fig. 1). Las cinéticas de crecimiento del consorcio bacteriano en presencia y ausencia de ROX fueron ajustadas con el algoritmo de Weibull (Zwietering et al. 1990), el cual fue el único modelo que presentó un R2 mayor a 0,907 en ambas situaciones (Fig. 2). Por otro lado, las velocidades de crecimiento $(\mathrm{k})$ del consorcio bacteriano incubado con ROX fue $0,00649 \mathrm{OD} \mathrm{h}^{-1} \mathrm{y}$ sin ROX fue 0,00471 OD $\mathrm{h}^{-1}$. Se observó una diferencia significativa entre ambas k (prueba t de Student, $\mathrm{P}=0,021$ ) y la velocidad de crecimiento en presencia de ROX fue 1,38 veces mayor que en ausencia del compuesto organoarsenical. Además, el modelo que mejor representó la cinética de degradación fue el algoritmo de Weibull (Zwietering et al. 1990) con un R2 de 0,961 donde la velocidad de degradación de ROX fue de $0,0250 \mathrm{OD} \mathrm{nm} \mathrm{h}^{-1}$.

EFECTO DE LA ROXARSONA SOBRE EL CONSORCIO BACTERIANO. Los perfiles de bandeo (OTUs) obtenidos por el DGGE posterior a $168 \mathrm{~h}$ de incubación del consorcio en presencia $\mathrm{y}$ ausencia de ROX, fueron analizados utilizando un escalamiento multidimensional (MDS) y los índices de diversidad de Shannon-Weaver $\left(H^{\prime}\right)$ y de equidad de Pielou $(J ')$. El porcentaje de similitud del consorcio bacteriano disminuyó de 70 a 50\% en presencia de ROX (Fig. 3). Por otro lado, el índice $H^{\prime}$ para el consorcio bacteriano incubado con ROX fue 1,67 y sin ROX fue 1,929, los cuales presentaron diferencia significativa (prueba $\mathrm{t}$ de Student, $\mathrm{P}=0,031)$. Además, el índice de equidad de Pielou estimado para el consorcio bacteriano incubado con $\operatorname{ROX}\left(J^{\prime}=\right.$ 0,9318 ) fue estadísticamente diferente (prueba t de Student, $\mathrm{P}=0,038)$ al estimado para el consorcio crecido sin $\mathrm{ROX}\left(J^{\prime}\right.$ $=0,8378$ ).

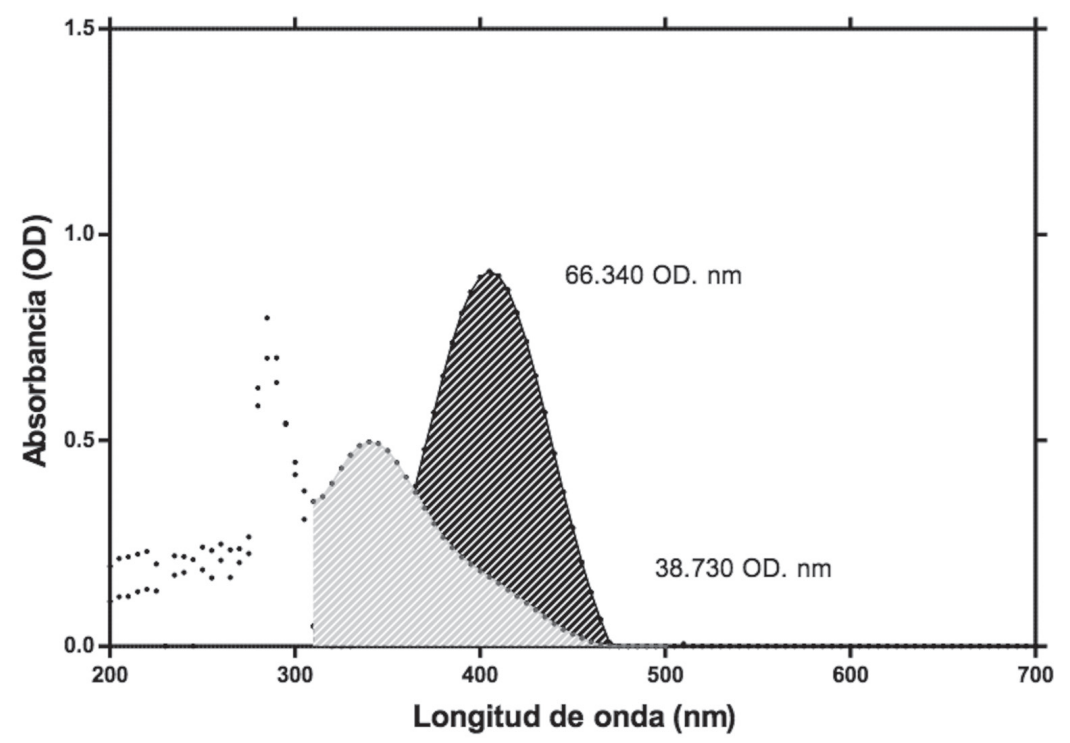

Figura 1. Degradación aeróbica de roxarsona por el consorcio bacteriano después de 7 días de incubación. Consorcio bacteriano crecido en R2A/2 (- $)$ y control negativo ( $\square$ ).

FIGURE 1. Aerobic degradation of roxarsone by bacterial consortium after 7 days of incubation. Bacterial consortium grown in R2A/2 (य) and negative control $(\square)$. 


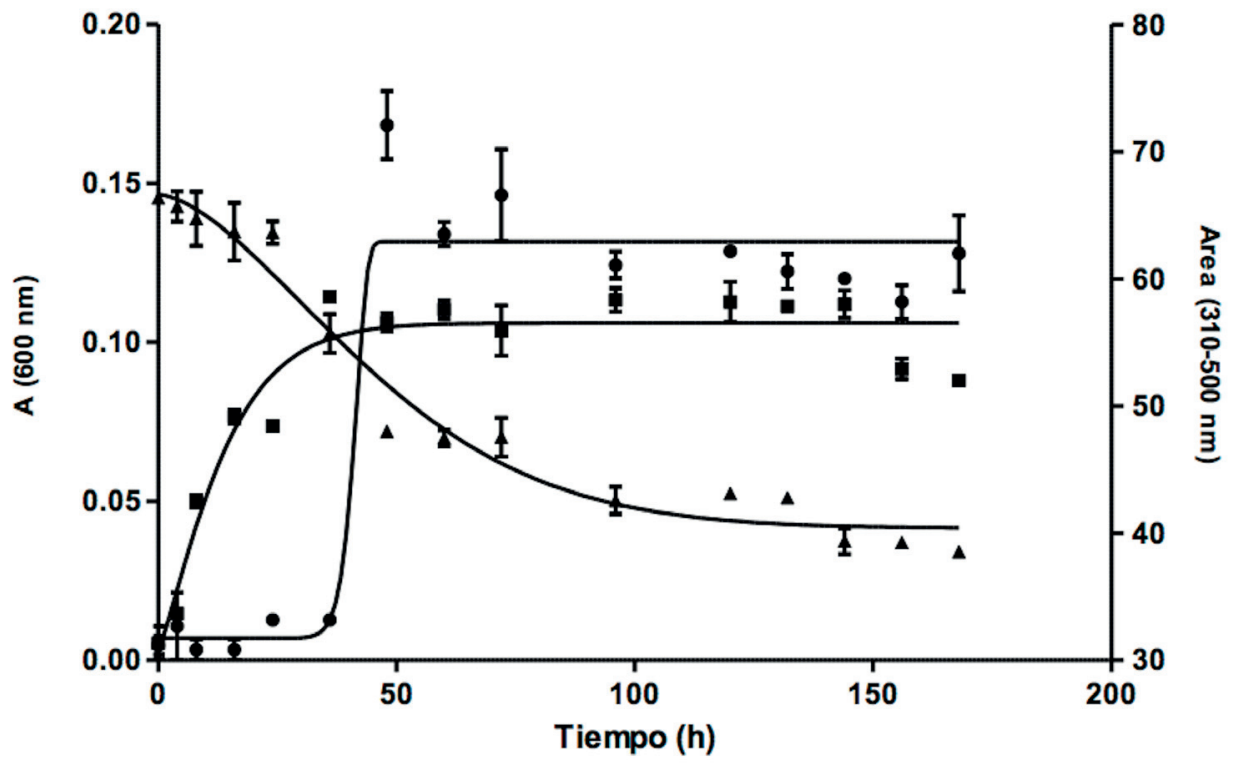

FiguRA 2. Cinéticas de crecimiento y degradación del consorcio bacteriano. La velocidad de degradación fue 0,0250 OD nm h-1 (R2= 0,961). La velocidad de crecimiento con y sin roxarsona fue 0,00649 OD h-1 (R2=0,907) y 0,00471 OD h-1 (R2=0,937), respectivamente. Las cinéticas de crecimiento y degradación fueron ajustadas con el algoritmo de Weibull. Degradación de roxarsona ( $\longrightarrow$ ), consorcio bacteriano sin roxarsona $(\rightarrow-)$ y consorcio bacteriano con roxarsona $(\bullet-)$.

FIGURE 2. Growth and degradation kinetics of bacterial consortium. Rate of roxarsone degradation was $0.0250 \mathrm{OD} \mathrm{nm}$ h-1 (R2=0.961). Growth rate of bacterial with and without roxarsone were 0.00649 OD h-1 (R2=0.907) y 0.00471 OD h-1 (R2=0.937), respectively. Kinetics and degradation were adjusted with Weibull growth. Degradation of roxarsone $(\bullet)$, bacterial consortium without roxarsone $(\hookrightarrow-)$ and bacterial consortium with roxarsone $(\rightarrow-)$.

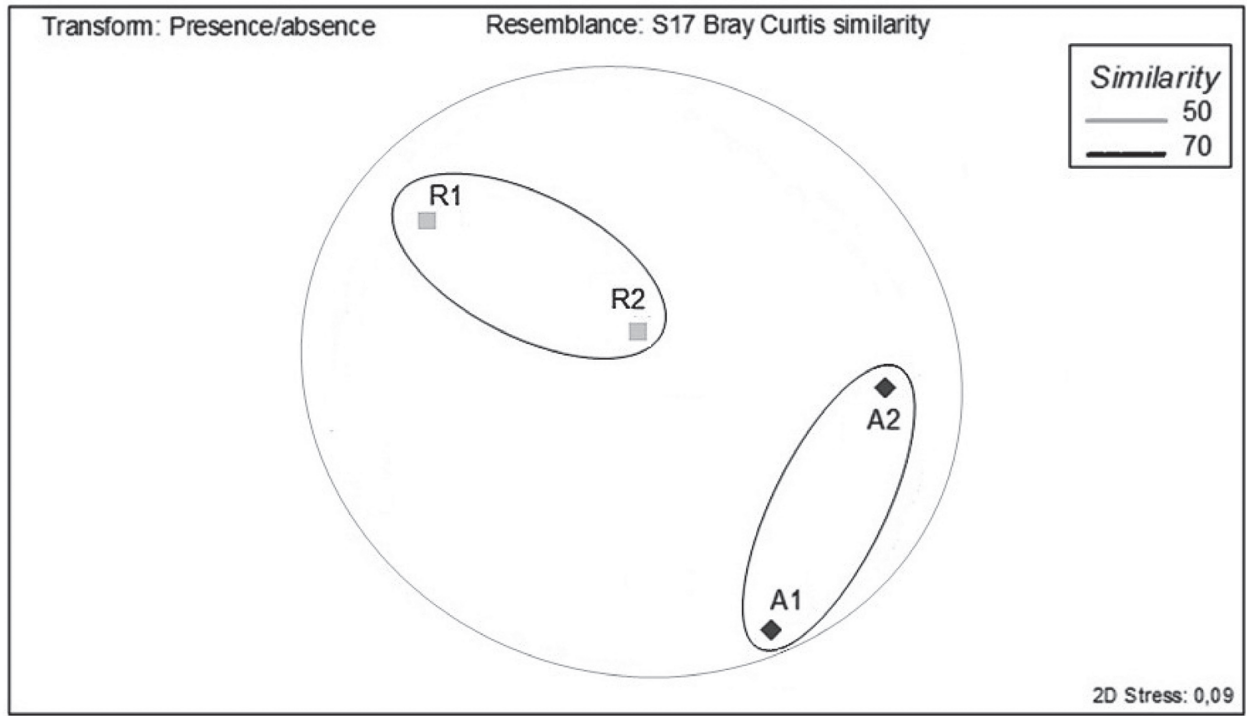

FIgURA 3. Diagrama de escalamiento multidimensional (MDS) de la matriz de datos del DGGE de fragmentos ADNr del consorcio bacteriano sin ROX (A1 y A2) y del consorcio bacteriano con ROX (R1 y R2). Los índices de similaridad fueron evaluados en porcentaje. Dos replicas fueron analizadas para cada consorcio.

FIGURE 3. Multidimensional scaling (MDS) of the DGGE data matrix of Eubacteria rDNA fragments from bacterial consortium without ROX (A1 and A2) and bacterial consortium with ROX (R1 and R2). Similarly index was evaluated for percentage. Two replicates were analyzed for each consortium. 
EFECTO DE LOS FACTORES ABIÓTICOS SOBRE LA DEGRADACIÓN DE LA ROXARSONA

El efecto de los factores abióticos en la biodegradación de ROX fue analizado determinando el porcentaje de degradación después de 120 h incubación en MQD.

Los porcentajes de degradación de ROX obtenidos a $15^{\circ} \mathrm{C}, 30^{\circ} \mathrm{C}$ y $37^{\circ} \mathrm{C}$ fueron respectivamente $34,3 \%, 47,8 \%$ y $59,7 \%$, además de significativamente diferentes entre sí (ANOVA, $\mathrm{P}=0,0001$ ) (Fig. 4A). La prueba de Tukey reveló que los resultados de biodegradación de $\mathrm{ROX}$ fueron mayores a $37^{\circ} \mathrm{C}$ en comparación a $15^{\circ} \mathrm{C}(\mathrm{P}=0,003)$ y $30^{\circ} \mathrm{C}$ $(\mathrm{P}=0,003)$. Además, el análisis utilizado demostró que la biodegradación de $\mathrm{ROX}$ a $30^{\circ} \mathrm{C}$ fue mayor que a $15^{\circ} \mathrm{C}$ $(\mathrm{P}=0,023)$.

El efecto del $\mathrm{pH}$ fue evaluado a $37^{\circ} \mathrm{C}$ como temperatura óptima de incubación. Los porcentajes de degradación de ROX obtenidos a $\mathrm{pH} 5,7$ y 9 fueron $35,9 \%, 62,3 \%$ y $6,38 \%$, respectivamente, cuyas diferencias fueron significativas (ANOVA, $\mathrm{P}=0,0001$ ) (Fig. 4B). La prueba de Tukey reveló que los resultados de biodegradación de ROX fueron mayores a $\mathrm{pH} 7$ en comparación con $\mathrm{pH} 5(\mathrm{P}=0,0046)$ y con $\mathrm{pH} 9(\mathrm{P}=0,0001)$. Además, éste análisis mostró que la biodegradación de $\mathrm{ROX}$ a $\mathrm{pH} 5$ fue mayor que a $\mathrm{pH} 9$ $(\mathrm{P}=0,0001)$.

El efecto de las fuentes de carbono fue evaluado a $37^{\circ} \mathrm{C}$ y pH 7 como condiciones óptimas de incubación. Los porcentajes de degradación de ROX obtenidos en presencia de acetato, glucosa, lactato y ausencia de fuente de carbono fueron $8,42 \%, 62,3 \%, 57,8 \%, y \quad 4,76 \%$ respectivamente, cuyas diferencias fueron significativas (ANOVA, $\mathrm{P}=0,0001$ ) (Fig. 4C). La prueba de Tukey reveló que los resultados de biodegradación de ROX no mostraron diferencia significativa entre el uso de glucosa o lactato $(\mathrm{P}=0,059)$. Además éste análisis demostró que la biodegradación de ROX fue mayor en presencia de glucosa en comparación con la presencia de acetato $(\mathrm{P}=0,0001)$ y sin fuente de carbono $(\mathrm{P}=0,0001)$. Igualmente, la prueba de Tukey reveló que la biodegradación es mayor en presencia de lactato en comparación con la presencia de acetato $(\mathrm{P}=0,0001)$ y sin fuente de carbono $(\mathrm{P}=0,0001)$. Por último, la presencia de acetato favoreció la degradación de ROX en comparación al consorcio control crecido sin fuente de carbono (prueba de Tukey, $\mathrm{P}=0,0001)$.
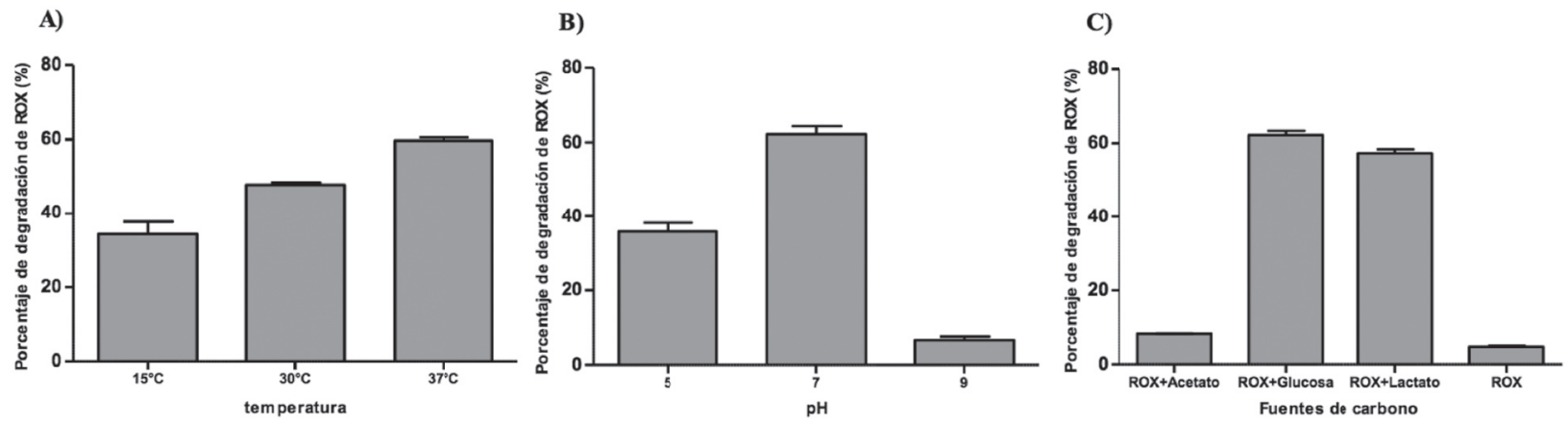

FiguRA 4. Efecto de factores abióticos en la degradación de roxarsona por un consorcio bacteriano después de 120 h de incubación. A) Temperatura. B) pH. C) Fuentes de carbono.

FIGURE 4. Effect of abiotic factors in the roxarsone degradation by bacterial consortium after $120 \mathrm{~h}$ of incubation. A) Temperature. B) pH. C) Carbon sources.

\section{DISCUSIÓN}

Desde una muestra de suelo agrícola se aisló un consorcio bacteriano capaz de degradar roxarsona en condiciones aeróbicas. Durante 7 días de incubación a $25^{\circ} \mathrm{C}$ (con agitación) se logró una reducción de un $41,6 \%$ de la ROX inicial en el medio R2A/2. Resultados similares fueron descritos por Garbarino et al. (2003) quienes demostraron procesos de transformación de ROX presente en el estiércol de ave de corral, pero bajo condiciones anaeróbicas. Estos procesos fueron principalmente bióticos y conducentes a la formación de arsénico inorgánico al cabo de 38 días de incubación. Además, Stolz et al. (2007) demostraron que una comunidad microbiana presente en muestras de suelo fue capaz de transformar hasta el $100 \%$ de roxarsona luego de 9 días de incubación, aunque también bajo condiciones anaeróbicas. Ninguno de estos autores reportó degradación de ROX en condiciones aeróbicas. 
Las cinéticas de crecimiento del consorcio bacteriano en presencia y ausencia de roxarsona muestran que la velocidad de crecimiento aumenta 1,38 veces en presencia del compuesto organoarsenical. Este incremento puede ser debido a la selección y posterior aumento exponencial de los grupos bacterianos que utilizan ROX como factor nutricional, tal como lo sugiere la diferencia entre los máximos valores $\mathrm{DO}_{600}$ en fase estacionaria para el consorcio adicionado con ROX frente al sin ROX (Fig. 2). El análisis MDS de los patrones de bandas indicó que los porcentajes de similitud del consorcio bacteriano son alterados por la presencia ROX (Fig. 3), sustentado la idea de la ROX como un agente selectivo sobre la comunidad bacteriana natural, generando un nuevo consorcio capaz de tolerar su toxicidad. Esto ha sido reportado por otros autores tanto para compuestos orgánicos tóxicos como para metales pesados (Campos et al. 2009; Campos et al. 2011; Silver \& Phung 2005). De hecho, los compuestos intermediarios generados durante la biotransformación de ROX son semejantes al ácido 4-hidroxi-3-amino fenil arsénico (HAPA), ácido monometilarsénico (MMA (V)), ácido monometilarsonoso (MMA (III)), ácido dimetilarsínico (DMA (V)), ácido dimetilarsinoso (DMA (III)), óxido de trimetilarsina (TMA (III)) y compuestos inorgánicos como arsenito (As (III)) y en mayor proporción arseniato (As (V)) (Andra et al. 2010; Cortinas et al. 2006; Stolz et al. 2007), pueden producir lisis celular por ser altamente tóxicos para la células. Un ejemplo es el As (V), un análogo de la estructura de fosfato que puede entrar a las células mediante transportadores de fosfato e inhibir la fosforilación oxidativa. En el presente estudio, el efecto tóxico de la ROX y/o de los compuestos derivados de su biotransformación queda de manifiesto tras observar una disminución significativa del índice diversidad de Shannon-Weaver ( $\left.\mathrm{H}^{\prime}\right)$ en presencia de ROX (prueba $\mathrm{T}$ de Student, $\mathrm{P}=0,031$ ), mientras que el índice de equidad de Pielou (J') aumentó en presencia del organoarsenical (prueba $\mathrm{T}$ de Student, $\mathrm{P}=0,038$ ). Estos resultados concuerdan con los estudios realizados por Collier et al. (2003) quienes analizaron la respuesta ecológica de la microbiota de porcino frente a diversos antimicrobianos promotores de crecimiento, entre ellos roxarsona, la cual produjo una disminución de la diversidad y un aumento de la homogeneidad en ésta microbiota. Por lo tanto, la metabolización de roxarsona afecta cinética y estructuralmente a las comunidades bacterianas nativas de un hábitat particular, generando consorcios bacterianos específicos capaces de degradar el compuesto.

La degradación de compuestos orgánicos resulta de la interacción de mecanismos bióticos y abióticos (Das et al. 2008). Los resultados obtenidos en el presente estudio demuestran que los factores abióticos afectan directamente la biotransformación de roxarsona. Esto queda demostrado al analizar los ensayos de degradación de ROX a diferentes temperaturas, con el mayor porcentaje de degradación $\left(59,7 \%\right.$ ) ocurriendo a $37^{\circ} \mathrm{C}$ (Fig. 4A). Este resultado fue consistente con el estudio realizado por Garbarino et al. (2003) quienes reportaron un bajo porcentaje (10\%) de degradación de $\mathrm{ROX}$ a 15 y $20^{\circ} \mathrm{C}$ en condiciones anaeróbicas, mientras que a $40^{\circ} \mathrm{C}$ observaron un capacidad degradativa muy superior (60\%). Lo anterior realza el efecto de la temperatura en la biotransformación de roxarsona ya que se sabe que el metabolismo celular es altamente dependiente de esta variable que influye, e.g., en la fluidez de la membrana citoplasmática para permitir un óptimo transporte de nutrientes o a la formación del gradiente de protones (Russell 2002).

Por otro lado, los resultados demostraron que a $\mathrm{pH}$ neutro la capacidad degradativa del consorcio fue mayor que a $\mathrm{pH}$ acido $(=5)$ y básico $(=9)$. La misma situación llevada al terreno implicaría que una microbiota nativa de suelos con pH 7 mostraría una buena capacidad de degradación de ROX, favoreciendo su movilización en el medioambiente. Estos resultados concuerdan con lo descrito por $\mathrm{Hu}$ et al. (2012), quienes indicaron que esta variable resulta ser uno de los factores químicos más importantes en los procesos de degradación, ya que para las sustancias químicas ionizables la variación del $\mathrm{pH}$ en la solución dará lugar a cambios en la especiación química, solubilidad y hidrofilicidad, lo que afectaría directamente a la biotransformación de roxarsona. Otro factor importante para la degradación de ROX es la disponibilidad de otras fuentes de carbono para la comunidad bacteriana. Las fuentes de carbono que promovieron mayores porcentajes de degradación de ROX fueron glucosa y lactato, mientras que en contraposición se situó el acetato (Fig. 4C). Esto concuerda con el estudio de Cortinas et al. (2006) quienes observaron un aumento significativo en la tasa de biotransformación ROX por hidrógeno, glucosa y lactato $(2,80,1,90$, y 1,80 veces, respectivamente) en referencia al control, pero una baja capacidad del acetato para estimular la degradación del organoarsenical. No obstante, dichos ensayos se realizaron en condiciones anaeróbicas. Es probable que la utilización aeróbica de glucosa sea principalmente catabólica, a través de la glicólisis y el transporte de electrones, aportando la energía necesaria para la ruptura de la molécula de ROX. Por otro lado, la oxidación de lactato ha sido descrita en varias especies bacterianas (Erwin \& Gotschlich 1993; Gao et al. 2012; Ingledew \& Poole 1984; Ma et al. 2007). $\mathrm{NAD}+$ lactato deshidrogenasas independientes (iLDHs) son las responsables de transformar el lactato a piruvato, donde los electrones removidos del lactato entran a la cadena transportadora de electrones para la producción de energía (Erwin \& Gotschlich 1993; Gao et al. 2012). Por lo tanto, glucosa y lactato serían donadores de electrones que además se comportarían como cosustratos metabólicos, ya que resultaron fundamentales para la degradación de ROX, 
situación que ya ha sido descrito por Stolz et al. (2007), previamente. En contraste, la utilización catabólica del acetato generaría menor poder energético que el resto de las fuente de carbono en condiciones aeróbicas, debido a que su oxidación se inhibe por sustrato como es el caso de las bacterias del género Acetobacter (Flanzy 2003). Es más probable que el acetato tenga un destino principalmente anabólico, como es el caso de las bacterias del genero Gluconobacter quienes son deficientes de $\alpha$-cetoglutarato deshidrogenasa y succinato deshidrogenasas (Kitos et al. 1958). Finalmente, no es menor la composición del medio químicamente definido (MQD) utilizado en los ensayos con los factores abióticos, que a diferencia de R2A/2, posee una mayor concentración de vitaminas y minerales los cuales participan como cofactores enzimáticos de las dioxigenasas (ya sea como Fe II o Fe III), además de NADPH y las flavoproteínas que son cofactores de oxigenasas, ambas enzimas fundamentales para degradación de compuestos aromáticos (Fuchs et al. 2011). El extracto de levadura presente en ambos medios, posiblemente actué también como un cosustrato de ROX en la situación de ausencia de fuente de carbono, lo cual explicaría el bajo porcentaje de degradación alcanzado.

Se puede concluir que la roxarsona y/o los compuestos derivados de su biotransformación alteran la estructura de las comunidades bacterianas nativas del suelo, dando paso al desarrollo de consorcios específicos capaces de tolerar y biotransformación este tipo de compuestos bajo condiciones aeróbicas. La biotransformación bacteriana de ROX sería favorecida por condiciones mesofílicas, un $\mathrm{pH}$ neutro y la presencia de cosustratos orgánicos de fácil metabolización. Este estudio se sitúa entre los primeros esfuerzos por entender los efectos de la roxarsona sobre las comunidades bacterianas aeróbicas de suelo así como los factores que influyen sobre su biodegradación, la cual está directamente relacionada con el impacto ambiental que pueden generar este tipo de compuestos tanto en suelos como en los sistemas acuáticos aledaños.

\section{AGRADECIMIENTOS}

Este trabajo fue financiado por el proyecto FONDECYT N ${ }^{\circ}$ 1110876. Proyecto VRID Nº123.036.040-1.0.

\section{BIBLIOGRAFÍA}

Andra, S. S., Makris, K. C., Quazi, S., Sarkar, D., Datta, R. \& BACH, S. B. 2010. Organocopper complexes during roxarsone degradation in wastewater lagoons. Environ. Sci. Pollut. Res. Int. 17: 1167-1173.

Arai, Y., Lanzirotti, A., Sutton, S., Davis, J. A. \& Sparks, D. L. 2003. Arsenic speciation and reactivity in poultry litter.
Environ. Sci. Technol. 37: 4083-4090.

Brosius, J., Palmer, M. L., Kennedy, P. J. \& Noller, H. F. 1978. Complete nucleotide sequence of a $16 \mathrm{~S}$ ribosomal RNA gene from Escherichia coli. Proc. Natl. Acad. Sci. U S A. 75: 4801-4805.

Campos, V. L., Escalante, G., Yañez, J., Zaror, C. A. \& Mondaca, M. A. 2009. Isolation of arsenite oxidizing bacteria from a natural biofilm associated to volcanic rocks of Atacama Desert, Chile. J. basic microbiol. 49: S93-S97.

Campos, V. L., Leon, C., Mondaca, M. A., Yanez, J. \& Zaror, C. 2011. Arsenic mobilization by epilithic bacterial communities associated with volcanic rocks from Camarones River, Atacama Desert, northern Chile. Arch. Environ. Contam. Toxicol. 61: 185-192.

Clarke, K. R. \& Gorley, R. N. 2001. PRIMER v5 (\& v6): User manual/tutorial, PRIMER-E, Plymouth UK.

Collier, C. T., Smiricky-Tuardes, M. R., Albin, D. M., Wubben, J. E., Gabert, V. M., Deplancke, B., Bane, D., Anderson, D. B. \& Gaskins, H. R. 2003. Molecular ecological analysis of porcine ileal microbiota responses to antimicrobial growth promoters. J. Anim. Sci. 81: 3035-3045.

Cortinas, I., Field, J. A., Kopplin, M., Garbarino, J. R., Gandolfi, A. J. \& Sierra-Alvarez, R. 2006. Anaerobic biotransformation of roxarsone and related N-substituted phenylarsonic acids. Environ. Sci. Technol. 40: 29512957.

DAs, P., PAL, R. \& ChowdhuRy, A. 2008. Influence of bioticabiotic factors on the degradation of novaluron in tropical soil. Int. J. Environ. Sci. Tech. 5: 425-429.

Erwin, A. L. \& Gotschlich, E. C. 1993. Oxidation of D-lactate and L-lactate by Neisseria meningitidis: purification and cloning of meningococcal D-lactate dehydrogenase. J. Bacteriol. 175: 6382-6391.

FlanZY, C. 2003. Enología: fundamentos científicos y tecnológicos. A. Madrid Vicente.

Fromin, N., Hamelin, J., Tarnawski, S., Roesti, D., JourdainMiserez, K., Forestier, N., Teyssier-Cuvelle, S., Gillet, F., Aragno, M. \& Rossi, P. 2002. Statistical analysis of denaturing gel electrophoresis (DGE) fingerprinting patterns. Environ. Microbiol. 4: 634-643.

Fuchs, G., Boll, M. \& Heider, J. 2011. Microbial degradation of aromatic compounds - from one strategy to four. Nat. Rev. Microbiol. 9: 803-816.

Gao, C., Hu, C., Zheng, Z., Ma, C., Jiang, T., Dou, P. I., Zhang, W., Che, B., WANG, Y. \& Lv, M. 2012. Lactate utilization is regulated by the FadR-type regulator LldR in Pseudomonas aeruginosa. J. Bacteriol. 194: 2687-2692.

Garbarino, J. R., Bednar, A. J., Rutherford, D. W., Beyer, R. S. \& Wershaw, R. L. 2003. Environmental fate of roxarsone in poultry litter. I. Degradation of roxarsone during composting. Environ. Sci. Technol. 37: 1509-1514.

Hu, J., Tong, Z., Hu, Z., Chen, G. \& Chen, T. 2012. Adsorption of roxarsone from aqueous solution by multi-walled carbon nanotubes. Journal of colloid and interface science. J. Colloid Interf. Sci. 337:355-361.

Ingledew, W. J. \& Poole, R. K. 1984. The respiratory chains of Escherichia coli. Microbiol. rev. 48: 222.

Jackson, B. P. \& Bertsch, P. M. 2001. Determination of arsenic speciation in poultry wastes by IC-ICP-MS. Environ. Sci. Technol. 35: 4868-4873. 
Jackson, B. P., Bertsch, P. M., Cabrera, M. L., Camberato, J. J., Seaman, J. C. \& Wood, C. W. 2003. Trace element speciation in poultry litter. J. Environ. Qual. 32: 535-540.

Kitos, P. A., Wang, C. H., Mohler, B. A., King, T. E. \& Cheldelin, V. H. 1958. Glucose and gluconate dissimilation in Acetobacter suboxydans. J. Biol. Chem. 233: 1295-1298.

Leon, C., Campos, V., Urrutia, R. \& Mondaca, M. A. 2012. Metabolic and molecular characterization of bacterial community associated to Patagonian Chilean oligotrophiclakes of quaternary glacial origin. World J. Microbiol. Biotechnol. 28: 1511-1521.

Ma, C., Gao, C., Qiu, J., HaO, J., Liu, W., Wang, A., Zhang, Y., WANG, M. \& XU, P. 2007. Membrane-bound L- and D-lactate dehydrogenase activities of a newly isolated Pseudomonas stutzeri strain. Appl. Microbiol. Biotechnol. 77: 91-98.

Muyzer, G., de Waal, E. C. \& Uitterlinden, A. G. 1993. Profiling of complex microbial populations by denaturing gradient gel electrophoresis analysis of polymerase chain reactionamplified genes coding for $16 \mathrm{~S}$ rRNA. Appl. Environ. Microbiol. 59: 695-700.

Nachman, K. E., Graham, J. P., Price, L. B. \& Silbergeld, E. K. 2005. Arsenic: a roadblock to potential animal waste management solutions. Environ. Health Perspect. 113:
1123-1124.

Nachman, K. E., Raber, G., Francesconi, K. A., Navas-Acien, A. \& Love, D. C. 2012. Arsenic species in poultry feather meal. Sci. Total Environ. 417-418: 183-188.

Pielou, E. C. 1975. Ecological diversity. Wiley, New York.

Russell, N. J. 2002. Bacterial membranes: the effects of chill storage and food processing. An overview. Int. J. Food Microbiol. 79: 27-34.

Shannon, C. E. \& Weaver, W. 1963. Mathematical theory of communication. University Illinois Press, Urbana.

Silbergeld, E. K. \& Nachman, K. 2008. The Environmental and Public Health Risks Associated with Arsenical Use in Animal Feeds. Ann. Ny. Acad. Sci. 1140: 346-357.

Silver, S. \& Phung, L. T. 2005. Genes and enzymes involved in bacterial oxidation and reduction of inorganic arsenic. Appl. Environ. Microbiol. 71: 599-608.

Stolz, J. F., Perera, E., Kilonzo, B., Kail, B., Crable, B., Fisher, E., Ranganathan, M., Wormer, L. \& Basu, P. 2007. Biotransformation of 3-nitro-4-hydroxybenzene arsonic acid (roxarsone) and release of inorganic arsenic by Clostridium species. Environ. Sci. Technol. 41: 818-823.

Zwietering, M. H., Jongenburger, I. L., Rombouts, F. M. \& Van't RIET, K. 1990. Modeling of the bacterial growth curve. Appl. Environ. Microbiol. 56: 1875-1881.

Recibido: 23.04.13

Aceptado: 25.09.13 